\title{
Performance Analysis of Machine Learning Algorithms Used for Web Based Phishing Detection.
}

\author{
${ }^{*}$ Shailendra Baliram Torane, \\ ${ }^{2}$ Dr. Narendra Shekokar.
}

${ }^{1}$ D. J. Sanghvi College of Engineering, Mumbai.

${ }^{2}$ Department of Computer Engineering, D. J. Sanghvi College of Engineering, Mumbai.

Email: ${ }^{1}$ shailendratorane93@ gmail.com, ${ }^{2}$ narendra.shekokar@djsce.ac.in

D.O.I - 10.51201/JUSST/21/05-187

http://doi.org/10.51201/JUSST/21/05187

\begin{abstract}
Phishing is a cybercrime technique in which the attacker creates a copy of genuine websites with the same color pattern, layout, font, and logo and with a domain name that matches with the realone. Then, broadcast this fake website through various online modes like emails and social media. The attacker creates lucrative offers or discounts to lure in people to click on the phishing link. Once the user clicks on this phishing link, they a re directed to the duplicate website that the attackerhad created. The user believes that it is the real website and enters his/her login details and other confidential data. This data is stored on the a ttacker's server thus giving him full a ccess to the victim's data. The phishing attack is ma inly targeted to collect confidential data of the victim. This data includes Username, Passwords, Bank details, security Credit card numbers etc. Machine Learning a lgorithms are being used widely in detecting phishing websites. This pa per shows performance analy sis of three Machine learning a lgorithms used for URL phishing detection. These algorithms are Extreme Learning Machine, Support Vector Machine and Naïve Bayes algorithm. The paper analyses these algorithms on the parameters of Accuracy, Precision, Recall, F1 score and Confusion matrix. The dataset includes 11,000 entries and 30 features from UC Irvine dataset repository. The literature survey shows how only importance is given to only one parameter i.e., Accura cy parameter when analyzing performance of the URL phishing detection algorithms. This pa per concludes on how Accuracy parameter does not show full picture on the overall performance of the URL phishing detection algorithms and a lso how Precision and Recall pa rameters a re very important in understanding the working of the se algorithms.
\end{abstract}

Key words: Phishing, Machine learning, cybersecurity, performance analysis, Phishing detection.

\section{INTRODUCTION}

With the advent of innovations in Mobile networks and Internet of Things (IoT) devices, Internet usage has increased enormously. A new genera tion of mobile network technology like 4G and $5 \mathrm{G}$ has provided highspeed internet to all its users. Therefore, a la rge no of new users is now using the internet. This has created a fa vorable situation for attackers to target new users.

A phishing a ttack is performed in many ways. Email phishing uses emails to broadcast a phishing URL to a larger number of audiences. Spear Phishing is a technique in which a specific group of people is targeted, for example, employees of an organization. This kind of Phishing a ttack needs moreknowledge about the target group. Whaling is similar to spear phishing but in Whaling the attacker masquerades as a senior employee of an organization and then directly target the attack on other important individuals in the orga nization with an aim to collect extremely sensitive information and then use it for criminal purposes. Smishing and Vishing a re other Phishing a ttacks in which the medium of communication is an SMS text messa ge and Voice call respectively. The SMS contains the same content a s the phishing email whereas in Vishing the attacker poses as a fraud investiga tor and asks for sensitive information on phone calls. 
Machine Learning algorithms are being used widely in detecting phishing websites. Machine learning is a field of computer science in which the algorithms are trained using a da taset and then made to perform theintended task. With training, the algorithm builds knowledge about the problem. The Algorithm then uses this knowledge and current inputs to the algorithm to make a particular decision. In this paper, three Machine Learning algorithms namely Ex treme Learning Machine, Naive Bayes and Support Vector Machine are implemented on a Dataset of 11,000Entries from UC Irvine website. The performance metrics calculated for this purpose are Accuracy, Precision, Recall, F1 Score, and Confusion Matrix.

\section{DATASET:}

The dataset used in this paper is 30 Fea tures dataset from UC Irvine Machine Learning Repository database. It has 11,000 binary en tries for all 30 features. These features include URL features and Web page features.

\begin{abstract}
A large da taset would train the Machine Learning algorithm on different cases a nd possibilities which would ultimately increase the chances of Phishing detection algorithm.
\end{abstract}

The performance metrics of machine learning algorithms are inter-related. This relation will help in combining the metrics instead of looking at them individually. The accuracy metrics is widely used to measure the performance of the Algorithms. The value of a ccuracy metric would change if less significant fea tures are selected for training the algorithm. The value of accuracy would change if da taset is changed even if we are using the same algorithm. The figure below shows the lists of 30 features from the dataset.

\begin{tabular}{|c|c|}
\hline Input (Features) & $\begin{array}{l}\text { Output } \\
\text { (Class) }\end{array}$ \\
\hline $\begin{array}{l}\text { 1.1. Address Bar based Features } \\
\text { 1.1.1. Using the IP Address } \\
\text { 1.1.2. Long URL to Hide the Suspicious Part } \\
\text { 1.1.3. Using URL Shortening Services "TinyURL" } \\
\text { 1.1.4. URL's having "@" Symbol } \\
\text { 1.1.5. Redirecting using "///" } \\
\text { 1.1.6. Adding Prefix or Suffix Separated by (-) to the } \\
\text { Domain } \\
\text { 1.1.7. Sub Domain and Multi Sub Domains } \\
\text { 1.1.8. HTTPS (Hyper Text Transfer Protocol with Secure } \\
\text { Sockets Layer) } \\
\text { 1.1.9. Domain Registration Length } \\
\text { 1.1.10. Favicon } \\
\text { 1.1.11. Using Non-Standard Port } \\
\text { Part of the Uxistence of "HTTPS" Token in the Domain }\end{array}$ & \multirow{4}{*}{$\begin{array}{l}-1 \text { Phishing } \\
1 \text { Legitimate }\end{array}$} \\
\hline $\begin{array}{l}\text { 1.2. Abnormal Based Features } \\
\text { 1.2.1.Request URL } \\
\text { 1.2.2. URL of Anchor } \\
\text { 1.2.3. Links in }<\text { Meta }>,<\text { Script }>\text { and }<\text { Link }>\text { tags } \\
\text { 1.2.4. Server Form Handler (SFH) } \\
\text { 1.2.5. Submitting Information to Email } \\
\text { 1.2.6.Abnormal URL }\end{array}$ & \\
\hline $\begin{array}{l}\text { 1.3. HTML and JavaScript based Features } \\
\text { 1.3.1. Website Forwarding } \\
\text { 1.3.2. Status Bar Customization } \\
\text { 1.3.3. Disabling Right Click } \\
\text { 1.3.4. Using Pop-up Window } \\
\text { 1.3.5. IFrame Redirection }\end{array}$ & \\
\hline $\begin{array}{l}\text { 1.4. Domain based Features } \\
\text { 1.4.1. Age of Domain } \\
\text { 1.4.2. DNS Record } \\
\text { 1.4.3. Website Traffic } \\
\text { 1.4.4. PageRank } \\
\text { 1.4.5. Google Index } \\
\text { 1.4.6. Number of Links Pointing to Page } \\
\text { 1.4.7. Statistical-Reports Based Feature }\end{array}$ & \\
\hline
\end{tabular}

Figure.5. Table of 30 Features in the dataset[1].

\section{IDENTIFICATION OF THE PROBLEM}

There are lot of datasets a nd a lgorithms which are being used for URL phishing detection. Thus, every time we cannot say that we can get better results if we are using certain dataset or only half of the dataset features or if we use this a lgorithm, we will be getting highest a ccuracy. The ability of URL phishing detection changes from algorithm to a lgorithm and from dataset to dataset. Based on the current a nalysis it is clear that feature selection plays an important role if we want our a lgorithms to identify all types of cases in the dataset. This paper uses a 30 features dataset from UC Irvine dataset repository which has features of Phishing URLs. There is more emphasis on the Accuracy parameter when determining the overall performance of the Phishing detection algorithms. But Accuracy parameter does not show many other cases are there in the dataset which are detected by the algorithm. Detection of these cases can help us understand the working of the a lgorithm in a better way. Thus, Precision and Recall come into picture. This study attempts to calculate performance metrics like Accuracy, Precision, Recall, F1 Score and Confusion Matrix for ELM, NB and SVM a lgorithms and analyze their performance in URL phishing detection. 


\section{ARCHITECTURE DIAGRAM}

The following architecture dia gram shows three algorithms namely Support Vector Machine, Naïve Bayes and Ex treme Learning Machine. It has a 30 fea tures dataset split into two: Training set and Testing set.

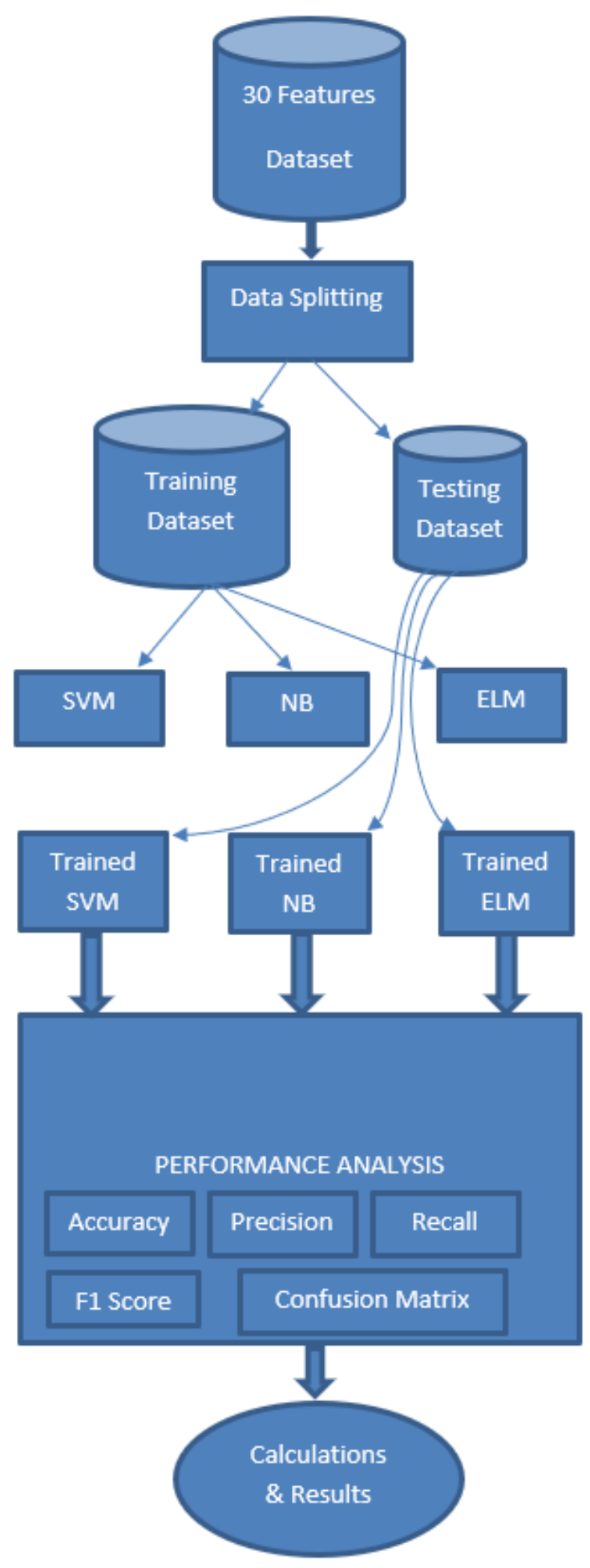

Performance metrics for machine lea rning algorithms a re calculated. Thesemetrics include Accuracy, Precision, Recall, F1 Score and Confusion Matrix.

\section{PERFORMANCE ANALYSIS}

\subsection{The PerformanceMetrics Calculated in this paper are as follow:}

\subsubsection{Classification Accuracy:}

It is the ratio of number of correct predictions to the total number of predictions made.

$$
\text { Acuracy }=\frac{\text { Number of Correct Preditions }}{\text { Total number of predictions made }}
$$

Figure. 1. Formula to calculate Accuracy.

\subsubsection{Precision:}

It is the number of correct positive results divided by the number of positive results predicted by the cla ssifier.

$$
\text { Precision }=\frac{\text { TruePositives }}{\text { TruePositives + FalsePositives }}
$$

Figure. 2. Formula to calculate Precision.

\subsubsection{Recall:}

It is the number of correct positive results divided by the number of all relev ant samples (all sa mples that should have been identified as positive).

$$
\text { Recall }=\frac{\text { TruePositives }}{\text { TruePositives + FalseNegatives }}
$$

Figure. 3. Formula to calculate Recall.

\subsubsection{F1 Score:}

F1 Score is used to measurea test's accuracy. The range for F1 Score is [0, 1]. It tells you how precise your cla ssifier is a well as how robust it is.

High precision but lower recall, gives you an ex tremely a ccurate, but it then misses a la rge number of in stances that are difficult to classify. The greater the F1 Score, the better is the performance of our model.

F1 Score tries to find the balance between precision and recall. 


$$
\mathrm{F} 1=2 * \frac{1}{\frac{1}{\text { Precision }}+\frac{1}{\text { Recall }}}
$$

Figure.4. Formula to calculate F1 Score.

\subsection{Confusion Matrix:}

Confusion Matrix as the name suggests gives us a matrix as output and describes the complete performance of the model.

There are 4 important terms:

- True Positives: The cases in which the model predicted YES and the actual out put was also YES.

- True Negatives: The cases in which the moedl predicted NO and the actual output was NO.

- False Positives: The cases in which themodel predicted YES and the actual output was NO.

- False Negatives: The cases in which themodel predicted NO and the actual output was YES.

Measuring an algorithm's efficiency is important beca use your choice of an algorithm for a given application often has a great impact. This paper shows comparison of a bove performance metrics for following algorithms:

- ExtremeMachine Learning.

- Support Vector Machine.

- Naïve Bayes.

The following shows calculation of performance metrics for Machine Learning Algorithms ELM, NB and SVM.

For each algorithm, Accuracy, Precision, Recall, F1 Score and Confusion Matrix is being calculated.

\subsection{Accuracy Analysis:}

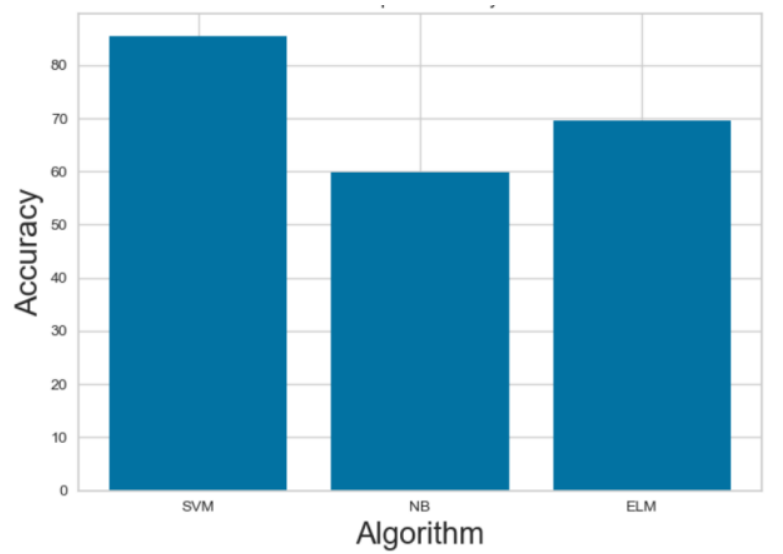

Figure.6. Accura cy Bar Graph for SVM, NB and ELM. The a bove graph shows the Accuracy achieved by SVM, NB and ELM. Accuracy is the number of accurately predicted outputout of total number of output predictions.

\begin{tabular}{|c|c|c|c|}
\hline Results & SVM & NB & ELM \\
\hline Accuracy & $85.43 \%$ & $59.90 \%$ & $70.14 \%$ \\
\hline
\end{tabular}

In the above table we can see that SVM performs best on the Accuracy parameter followed by ELM and then NB. The Accuracy of the ELM algorithm changes with the number of neurons involved in the execution.

\subsection{Confusion Matrix Analysis:}

\subsubsection{Confusion Matrix of Support Vector Machine Algorithm:}

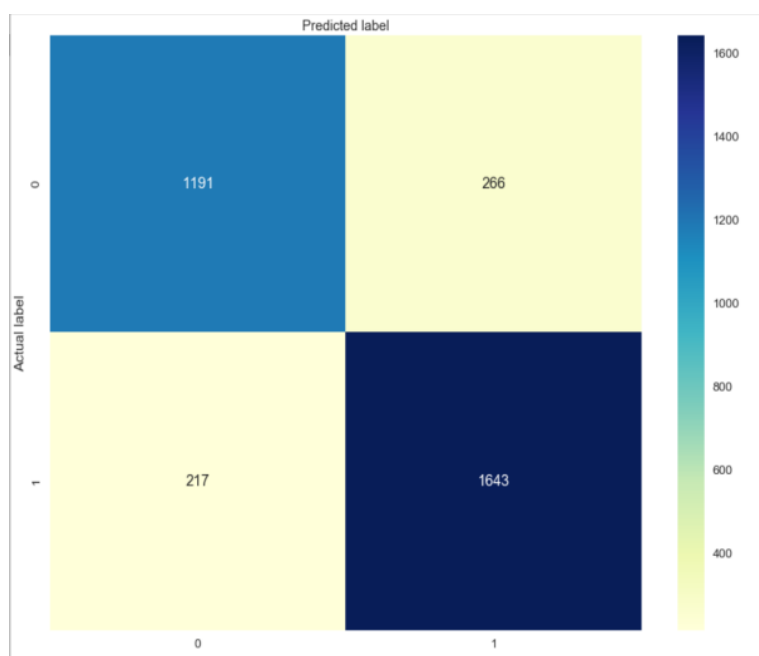

Figure.7. Confusion Matrix of SVM.

The above confusion matrix is calculated for 3317 entries. 


\begin{tabular}{|l|l|}
\hline True Negative $=1191$ & False Positive $=266$ \\
\hline False Negative $=217$ & True Positive $=1643$ \\
\hline
\end{tabular}

With highest accuracy achieved, SVM has the high value for True Negative and True Positive in the confusion matrix. Total correct predicts are 2834 out of 3317 predictions. But we can see that the value of Fa lse Nega tive is 217 which means that out of 3317 en tries, SVM algorithm predicted 217 Phishing URLs a s NonPhishing URLs.

\subsubsection{Conf usion Matrix of Naïve Bayes Algorithm:}

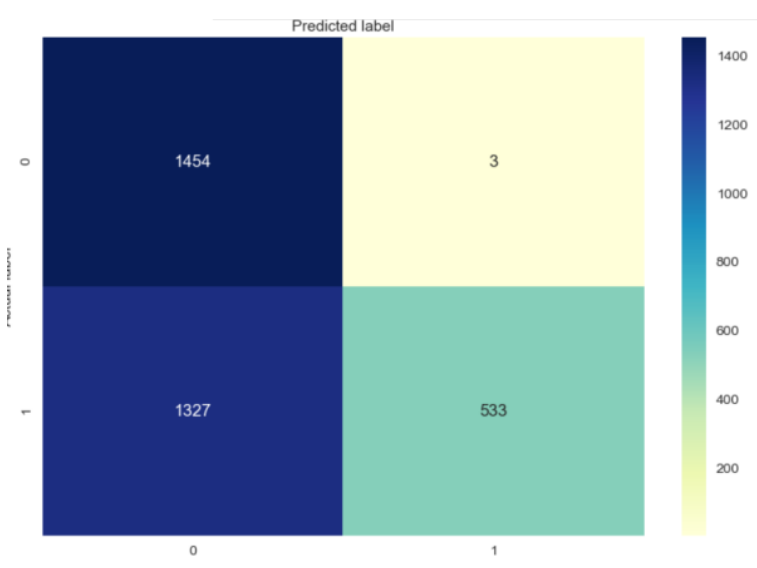

Figure.8. Confusion Matrix of NB.

\begin{tabular}{|l|l|}
\hline True Negative $=1454$ & False Positive $=3$ \\
\hline False Negative $=1327$ & True Positive $=533$ \\
\hline
\end{tabular}

Naive Bayes algorithm a chieved highest value for Fa lse Negative thus lowering the overall accuracy of the algorithm. The Fa lse Positive value is lowest a mong all the three algorithms. That means the Naïve Bayes algorithm was predicting fewer wrong positives as compared to Naïve Bayes and ELM.

\subsubsection{Conf usion Matrix of Extreme Learning Machine Algorithm:}

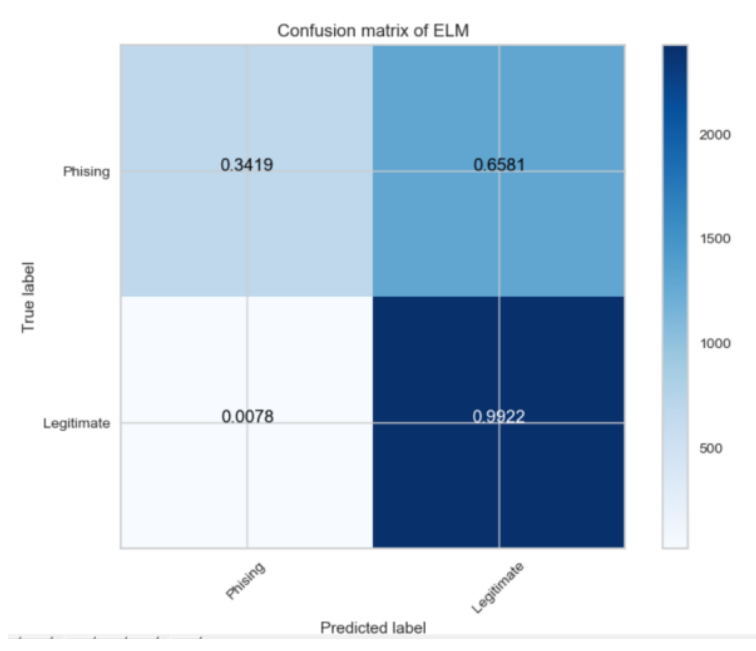

Figure.9. Confusion Matrix of ELM.

As we know, ELM is an Artificial Neural network a lgorithm and it has an analytical learning process. The performance of the ELM algorithm a lso depends on the number of neurons involved in the operation.

Out of total values that are a ctually phishing, $34.19 \%$ were predicted correctly as Phishing and $65.81 \%$ were predicted wrongly as Non phishing.

Out of total values that are actually Non-Phishing, $0.78 \%$ were predicted wrongly a s Phishing and $99.22 \%$ were predicted correctly as Non-Phishing.

\begin{tabular}{|l|l|}
\hline True Positives $=0.3419$ & False Positive $=0.6581$ \\
\hline False Negative $=0.0078$ & True Negative $=0.9922$ \\
\hline
\end{tabular}

\subsection{Classification Report Analysis}

The cla ssification report shows us following four parameters:
1. Precision
2. Recall
3. F1-Score

To examine the overall working of a model we need to look in to both precision and recall values.

\subsubsection{Classification Report of SVM:}

\begin{tabular}{|rrrrr} 
Classifiaction Report of SVC & & \\
& precision & recall & fl-score & support \\
Yes & 0.85 & 0.82 & 0.83 & 1457 \\
No & 0.86 & 0.88 & 0.87 & 1860 \\
& & & & \\
accuracy & & & 0.85 & 3317 \\
macro avg & 0.85 & 0.85 & 0.85 & 3317 \\
weighted avg & 0.85 & 0.85 & 0.85 & 3317
\end{tabular}


Figure.10. Classification Report of SVM.

The precision value for Yes is 0.85 , that means out of total predictions which were made as Phishing (Yes), $85 \%$ were precisely predicted a $\mathrm{Phishing}$.

The Precision value for No is 0.86 , that means out of total predictions which were made as Non-Phishing (No), $86 \%$ were precisely predicted as Non-Phishing.

The recall va lue for Yes is 0.82 , that means out of total no of entries that were a ctually Phishing (Yes), $82 \%$ were correctly recalled as phishing.

The recallvalue for No is 0.88 , that tmeans out of total no of entries that were actually Non-Phishing, $88 \%$ were correctly recalled as Non-Phishing.

F1 score calculates the Harmonic mean between Precision and Recall values. The high F1 score indicates that the model has high Precision and Recall values.

The F1 score for Yes is 0.83 which is the Harmonic mean of Yes for Precision and Yes value for Recall i.e., 0.85 and 0.82 respectively.

The F1 sore for No is 0.87 which is the Ha monic Mean of No for Precision and No for Recall i.e., 0.86 and 0.88 respectively.

\subsubsection{Classification Report of NB:}

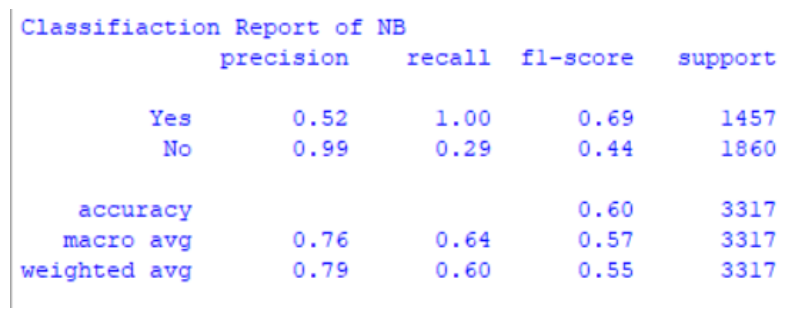

Figure.11. Classification Report of NB.

The a ccuracy valuefor NB is lowest a mong three algorithms thus it has lowervalues Precision and Recall.

The va lue of Yes for Precision is 0.52 , that means only $52 \%$ of total predictions made as Phishing (Yes) were correctly predicted as Phishing (Yes).

The value of No for Precision is rounded up to 0.99 , that means $99 \%$ of total predictions made as Non-
Phishing (No) were precisely predicted as Non-Phishing (No).

The value of Yes for Recall is rounded up to 1.0, that means $99 \%$ of predictions which were made as Phishing (Yes) were precisely predicted out of total actual predictions as Phishing (Yes).

The value of No for Recall is 0.29 , that means out of total actual Non-Phishing (No) predictions, $29 \%$ of predictions which were made as Non-Phishing were recalled correctly.

The F1 score for Yes is 0.69 which is the harmonic mean of Yes for Precision and Yes for Recall i.e., 0.52 and 1.00 respectively.

The F1 score for No is 0.44 which is the Harmonic mean of No for Precision and No for Recall i.e., 0.99 and 0.29 respectively.

\subsubsection{Classification Report of ELM:}

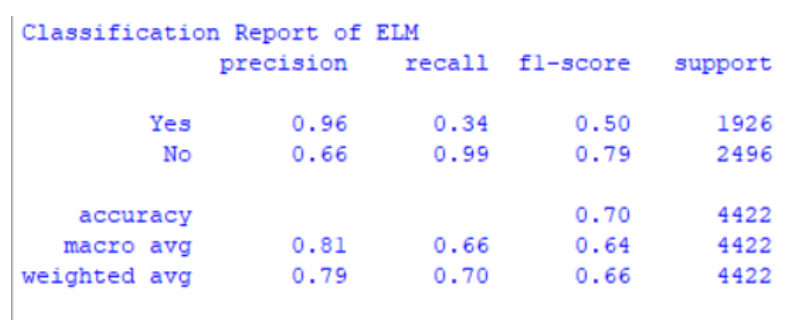

Figure.12. Cla ssification Report of ELM.

The Precision va lue for yes is 0.96 , that means out of all the predictions made as Phishing (Yes), $96 \%$ were correctly predicted.

The Precision va lue for No is 0.66 , that means out of all the predictions made a s Non-Phishing (No) only $66 \%$ were correct predictions as Non-Phishing.

The Recall value for Yes is 0.34 , that means out of total no of entries that were actually Phishing (Yes) only $34 \%$ were correctly recalled as phishing.

The Recall value for No is 0.99 , that means out of total no of entries that were actually Non-Phishing (No), $99 \%$ were correctly recalled as phishing.

The $\mathrm{F} 1$ sore for Yes is 0.50 which is the harmonic mean of Yes for Precision and Yes for Recall i.e., 0.96 and 0.34 respectively.

The F1 score for No is 0.79 which is the harmonic mean of No for Precision and No for Recall i.e., 0.66 and 0.99 respectively. 


\section{CONCLUSION}

In this pa per we did performance analysis of three algorithms namely Support Vector Machine, Naive Bayes and ExtremeLearning Machine. The algorithms are a nalyzed on four parameters they are Accuracy, Precision, Recall andF1 Score.

SVM achieved highest a ccuracy but ranked $2^{\text {nd }}$ in term s of predicting Phishing URLs after ELM algorithm. ELM algorithm has thehighest Precision for predicting URLs a s Phishing URLs but ranks $2^{\text {nd }}$ in the race of achieving Accuracy. Naïve Bayes algorithm ranks third in the race of achieving Accuracy after SVM and ELM, but has the highest recall value for predicting URLs as Phishing. Even though ELM ranks $2^{\text {nd }}$ in terms of Accura cy, it ranks $3^{\text {rd }}$ in term s for Recalling URLs as Phishing.

Every algorithm ranks different when analyzed on different parameters. Not necessary that algorithm with highest accuracy will also rank high in terms of other parameters like Precision and Recall.

The Accuracy parameter is not enough when we a re analyzing performance of models identifying URL phishing and thus need to focus on Recall and Precision parameter a s well. The Recall parameter need to be focused more as it detects False Negatives i.e., predicting Phishing URL as Non-Phishing. Thus, achieving high recall for URL phishing detection model is very important.

\section{FUTURE WORK}

In this pa per, performance analysis is done on three algorithm s using 30 features dataset. In future, out of these 30 features, best minimum features will be identified using Forward selection technique and then performance a nalysis will be done on it. Also, a hybrid approach for URL phishing detection will be implemented with 30 features and a lso with best minimum features identified ea rlier and its performance analysis will be done based on parameters like Accuracy, Precision, Recall and F1 Score.

\section{REFERENCES}

[1] Yasin Sönmez, Türker Tuncer, Hüseyin Gökal, Engin Avci, "Phishing Web Sites Features

Classification Based on Extreme Learning Ma chine",
2018 6th International Symposium on DigitalForensic and Security (ISDFS).

[2] T. Peng, I. Harris, and Y. Sawa, "Detecting Phishing Atta cks Using Natural La nguage processing and Machine Learning", Proc. $-12^{\text {th }}$ OEEE Int, Conf. Semant.Comput. ICSC2018, vol. 2018-Janua, pp. 300301, 2018.

[3] Aniruddha Joshi, Tanuja Pattanshetti "Phishing Atta ck Detection using Feature Selection Techniques". Proeedings on International Conference on Communication and Information processing (ICCIP) 12 Jul 2019 Last revised: 30 Sept 2019.

[4] Bhagyashree E. Sananse, Ta nuja K. Sarode "Phishing URL Detection: A Machine Learning and Web Mining-based Approach". International Journal of Computer Applications (0975 - 8887) Volume 123 No.13, August 2015.

[5] L. MacHad0 and J. Gadge, "Phishing Sites Detection Based on C4.5 Decision Tree Algorithm," in 2017 International Conference on Computing, Communication, Control and Automation, ICCUBEA 2017, 2018, pp. 1-5.

[6] S. Parekh, D. Parikh, S. Kotak, and P. S. Sankhe, "A New Method for Detection of Phishing Websites: URL Detection," in 2018 Second International Conference on Inventive Communication and Computational Technologies (ICICCT), 2018, vol. O, no. Icicct, pp. 949-952.

[7] Anjum N Shaikh, Antesar M Shabut, M. A. Hossain, "A Literature Review On Phishing Crime, Prevention Review and Investigation of Ga ps", $201610^{\text {th }}$ Internation conferences on Software, knowledge, Information Managementand Applications (SKIMA).

[8] Joby James, Sa ndhya L, Ciza Thomas, "Detection of Phishing URLs Using Ma chine Learning Techniques", 2013 International conference on Control Communication and Computing.

[9] Sneha Mande, Prof. D.S.Thosar, "Detection Of Phishing Web Sites Based On Extreme Machine Learning",Vol-4 Issue-62018, IJARIIE-ISSN(O)-23954396. 